\title{
Message from the Editor
}

\author{
J. Bogousslavsky, Lausanne
}

It is the policy of the journal to rotate the members of the Editorial Board every now and then and I would like to thank the following people for their great efforts over the past years:

\section{Loeb, Genova \\ E. Lugaresi, Bologna \\ J.H.J. Wokke, Utrecht}

I would also like to welcome the new members of the Editorial Board and thank them for agreeing to support European Neurology:

\section{J. Berciano, Santander \\ G.L. Lenzi, Rome \\ J. Sander, Gerrards Cross}

The following list of names are those of ad hoc reviewers, to whom I would also like to express my gratitude (October 1998 - October 1999):

P. Aebischer, Lausanne, Switzerland

A. Aguzzi, Zürich, Switzerland

A. Albanese, Lausanne, Switzerland

S. Anderson, Iowa City, Iowa, USA

G.M. Annoni, Geneva, Switzerland

Z. Argov, Jerusalem, Israel/Montreal, Canada
H. Barnett, London, Ont., Canada

C. Bassetti, Bern, Switzerland

R.W. Baumgartner, Zürich, Switzerland

S.R. Benbadis, Tampa, Fla., USA

J. Bille, Lausanne, Switzerland

J. Biollaz, Lausanne, Switzerland

T.D. Bird, Seattle, Wash., USA

W.T. Blume, London, Ont., Canada

N. Bornstein, Ramat Aviv, Israel

F.-X. Borruat, Lausanne, Switzerland

F.T. Bosman, Lausanne, Switzerland

C. Bouras, Chêne-Bourg, Switzerland

A. Brice, Paris, France

W. Brown, Boston, Mass., USA

J.C.M. Brust, New York, N.Y., USA

J.M. Burgunder, Bern, Switzerland

L.R. Caplan, Boston, Mass., USA

R. Chiolero, Lausanne, Switzerland

D. Chofflon, Geneva, Switzerland

M. Clanet, Toulouse, France

S. Clarke, Lausanne, Switzerland

G. Comi, Milan, Italy

A. Compston, Cambridge, UK

C. Confavreux, Lyon, France

L. Corlett, Oxford, UK

A. Culebras, Syracuse, N.Y., USA

J. de Reuck, Gent, Belgium

N. de Tribolet, Lausanne, Switzerland

J.D. Degos, Créteil, France

\section{KARGER}

Fax + 41613061234

E-Mail karger@karger.ch

www. karger.com (c) 2000 S. Karger AG, Basel

Accessible online at: www. karger.com/journals/ene 
P.J. Delwaide, Liège, Belgium

G. Devuyst, Lausanne, Switzerland

J.M. Dichgans, Tübingen, Germany

M. Dichgans, Munich, Germany

S. DiDonato, Milan, Italy

H.-C. Diener, Essen, Germany

A. Engel, Rochester, Minn., USA

L. Findley, London, UK

M. Fisher, Worcester, Mass., USA

P. Francioli, Lausanne, Switzerland

R.C. Gaillard, Lausanne, Switzerland

P. Genton, Marseille, France

J. Ghika, Lausanne, Switzerland

M. Giroud, Dijon, France

J.H. Growdon, Boston, Mass., USA

K. Hess, Zürich, Switzerland

J. Hildebrand, Brussels, Belgium

C. Hock, Zürich, Switzerland

P. Jallon, Geneva, Switzerland

L. Kappos, Basel, Switzerland

M. Kawamura, Tokyo, Japan

J. Kesselring, Valens, Switzerland

J. Kimura, Iowa City, Iowa, USA

T. Kuntzer, Lausanne, Switzerland

T. Landis, Geneva, Switzerland

J. Le Floch-Rohr, Geneva, Switzerland

K.L. Leenders, Groningen, The Netherlands

N. Leigh, London, UK

D. Leys, Lille, France

J.M. Léger, Paris, France

C. Loeb, Genova, Italy

O.L. Lopez, Pittsburgh, Pa., USA

H.P. Ludin, St. Gallen, Switzerland

O. Lyon-Caen, Paris, France

P.A. Lyrer, Basel, Switzerland

P. Maeder, Lausanne, Switzerland

P.J. Magistretti, Lausanne, Switzerland

J. Mathis, Bern, Switzerland
O. Meienberg, Binningen, Switzerland

R.A. Meuli, Lausanne, Switzerland

C. Miller Fisher, Boston, Mass., USA

H.W. Moser, Baltimore, Md., USA

B. Nater, Lausanne, Switzerland

H.-W. Pfister, Munich, Germany

C. Pierrot-Deseilligny, Paris, France

M. Reichhart, Lausanne, Switzerland

H. Reichmann, Dresden, Germany

E.B. Ringelstein, Münster, Germany

A.B. Safran, Geneva, Switzerland

G. Said, Le Kremlin Bicêtre, France

P. Scheltens, Amsterdam, The Netherlands

M. Schluep, Lausanne, Switzerland

D.F. Schorderet, Lausanne, Switzerland

M. Seek, Geneva, Switzerland

S.D. Shorvon, London, UK

V. Silani, Milan, Italy

C.J.M. Sindic, Bruxelles, Belgium

F. Spertini, Lausanne, Switzerland

A.J. Steck, Basel, Switzerland

M. Sturzenegger, Bern, Switzerland

E. Tournier-Lasserve, Paris, France

F.G.A. Van Der Meché, Rotterdam, The Netherlands

J.J. Van Hilten, Leiden, The Netherlands

M. Vidailhet, Paris, France

J.G. Villemure, Lausanne, Switzerland

A. Vincent, Oxford, UK

F. Vingerhoets, Lausanne, Switzerland

J.-P. G. Vonsattel, Charlestown, Mass. USA

P. Vuadens, Sion, Switzerland

H.G. Wieser, Zürich, Switzerland

J.H.J. Wokke, Utrecht, The Netherlands

A. Yamadori, Sendai, Japan

Y. Yonekawa, Zürich, Switzerland

D. Zegers de Beyl, Brussels, Belgium

D. Zochodne, Calgary, Canada 